\title{
Fast and automatic microseismic phase-arrival detection and denoising by pattern recognition and reduced-rank filtering
}

\author{
Danilo Velis ${ }^{1}$, Juan I. Sabbione ${ }^{2}$, and Mauricio D. Sacchi ${ }^{2}$
}

\begin{abstract}
We have developed a fast method that allowed us to automatically detect and denoise microseismic phase arrivals from $3 \mathrm{C}$ multichannel data. The method is a two-step process. First, the detection is carried out by means of a pattern recognition strategy that seeks plausible hyperbolic phase arrivals immersed in noisy $3 \mathrm{C}$ multichannel data. Then, the microseismic phase arrivals are denoised and reconstructed using a reduced-rank approximation of the singular value decomposition of the data along the detected phase arrivals in the context of a deflation procedure that took into account multiple arrivals and/or phases. For the detection, we have defined an objective function that measured the energy and coherence of a potential microseismic phase arrival along an apex-shifted hyperbolic search window.
\end{abstract}

The objective function, which was maximized using very fast simulated annealing, was based on the energy of the average signal and depended on the source position, receivers geometry, and velocity. In practice, the detection process did not require any a priori velocity model, leading to a fast algorithm that can be used in real time, even when the underlying velocity model was not constant. The reduced-rank filtering coupled with a crosscorrelation-based synchronization strategy allowed us to extract the most representative waveform for all the individual traces. Tests using synthetic and field data have determined the reliability and effectiveness of the proposed method for the accurate detection and denoising of $3 \mathrm{C}$ multichannel microseismic events under noisy conditions. Two confidence indicators to assess the presence of an actual phase arrival and the reliability of the denoised individual wave arrivals were also developed.

\section{INTRODUCTION}

The study of microseismic data has become an essential tool in many geoscience fields, such as oil reservoir geophysics (Maxwell et al., 2010; Kendall et al., 2011), mining engineering (Sun et al., 2012), and $\mathrm{CO}_{2}$ sequestration (Zhou et al., 2010). In hydraulic fracturing, microseismicity studies permit the characterization and monitoring of the reservoir dynamics to optimize the production and the fluid injection process itself. Often, real-time functionality is needed to control the fluid injection in the field for economic and safety reasons. Besides, because the number of events is usually large and the signal-to-noise ratio $(\mathrm{S} / \mathrm{N})$ is generally very low (especially when receivers are located at the surface), fast, automated, and robust detection algorithms are required for most applications (Zimmer and Jin, 2011). Furthermore, inadequate array coverage and uncertainties in the P- and S-wave underlying velocity models complicate the processing of the data (Eisner et al., 2010).

A basic workflow includes processes such as denoising, detection, picking, and localization (van der Baan et al., 2013). Generally, microseismic events are detected (and located) by means of grid-search algorithms (Maxwell, 2014). These techniques are very effective and accurate but computationally intensive when dealing with large 3D or 4D grids (Lagos et al., 2014). Other methods require picking before carrying out localization, and others are able to locate the microseisms without any picking at all (Gharti et al., 2010). As for detection, some techniques are based on the analysis of the changes of a certain attribute along individual channels, such as trace energy (Munro, 2004), its absolute value (Chen and Stewart, 2006), or a combination of short time average over long time average (STA/LTA) and the wavelet transform (Vera Rodriguez,

\footnotetext{
Manuscript received by the Editor 28 November 2014; revised manuscript received 14 May 2015; published online 27 July 2015.

${ }^{1}$ Universidad Nacional de La Plata, Facultad de Ciencias Astronómicas y Geofísicas, La Plata, Argentina and CONICET, Argentina. E-mail: velis@fcaglp.unlp.edu.ar.

${ }^{2}$ University of Alberta, Department of Physics and Signal Analysis and Imaging Group, Edmonton, Alberta, Canada. E-mail: jsabbione@ fcaglp.unlp.edu.ar; msacchi@ualberta.ca.

(C) 2015 Society of Exploration Geophysicists. All rights reserved.
} 
2011), to name a few. Nevertheless, any single-channel event detection algorithm is prone to fail at some point whenever the S/ $\mathrm{N}$ is very poor because not all data information is taken into account simultaneously. Thus, the key of a reliable event-detection algorithm relies on its ability to incorporate all the multichannel information at once. Sabbione and Velis (2013) follow this strategy and devise a simple method based on single-trace phase pickers borrowed from global seismology that uses all channels simultaneously. Zimmer and Jin (2011) and Gharti et al. (2010), for example, describe the so-called migration-based approach. This approach uses the information contained in a kind of stack of the input channels and does not require any trace-by-trace preprocessing for picking the individual arrivals. Likewise, Radon-based techniques (Sabbione et al., 2013, 2015) also rely on the information contained in all the channels simultaneously.

In this work, we present a fast two-stage method that does not require the knowledge of the underlying velocity model because an effective constant velocity is obtained as a by-product. First, we accomplish the detection by solving a pattern recognition problem very efficiently using very fast simulated annealing (VFSA) (Velis et al., 2013). This leads to a fully automatic and reliable microseismic phase-arrival detection algorithm that includes a deflation process used to deal with multiple phases and/or arrivals and a confidence indicator to assess the significance of the detections. Second, we denoise and reconstruct the detected phase arrivals using a singular value decomposition (SVD)-based reduced-rank filtering approach, which leads to consistent waveforms that preserve ampli-
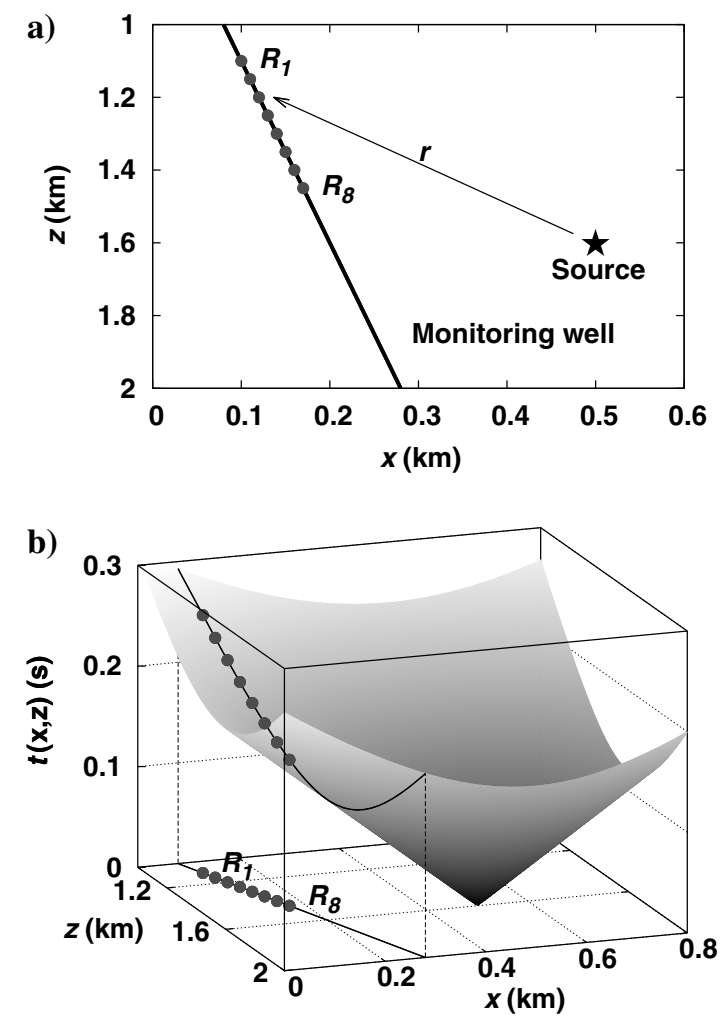

Figure 1. (a) Typical 2D downhole microseismic monitoring scenario and (b) the cone represents the arrival times expressed in equation 3. For a linear monitoring well, arrivals are aligned along an apex-shifted hyperbola. The $R_{1}$ and $R_{8}$ denote the first and eighth receivers, respectively. tudes throughout all available channels and components. As we show in the next sections, under certain assumptions, microseismic phase arrivals are characterized by coherent arrivals along hyperbolic patterns. Then, imperfect alignments are tolerated by means of a crosscorrelation-based synchronization process that is applied before the filtering. In addition, we provide another confidence indicator to assess the significance of the reconstructed phase arrivals on a trace-by-trace basis. By means of examples, we show that the proposed technique behaves very effectively and accurately when applied to moderate to high-S/N synthetic and field data sets, and reasonably well when applied to very noisy data sets. Overall, the detection is robust in the presence of random noise and isolated traces with useless information, the derived traveltimes are accurate, and the reconstructed waveforms are consistent with the observed data, even when the underlying velocity model is unknown.

\section{THEORY}

Consider a 2D typical downhole microseismic monitoring scenario as shown in Figure 1a. Assuming a constant velocity medium, the arrival time at the receiver $(x, z)$ due to a microseism originated at $\left(x_{s}, z_{s}\right)$ can be expressed by

$$
t(x, z)=t_{0}+\frac{r(x, z)}{v},
$$

where

$$
r(x, z)=\sqrt{\left(x-x_{s}\right)^{2}+\left(z-z_{s}\right)^{2}}
$$

is the source-receiver distance, $v$ is the $\mathrm{P}$ - or $\mathrm{S}$-wave velocity, and $t_{0}$ is the occurrence time of the event measured relatively to the start time of the recorded seismic traces.

\section{Cones and hyperbolas}

Equation 1 can be rewritten as

$$
\frac{\left(x-x_{s}\right)^{2}}{v^{2}}+\frac{\left(z-z_{s}\right)^{2}}{v^{2}}=\left(t-t_{0}\right)^{2}
$$

which is the equation of a right circular cone in $(x, z, t)$ centered at $\left(x_{s}, z_{s}, t_{0}\right)$. Figure $1 \mathrm{~b}$ shows part of this quadratic surface for $\left(x_{s}, z_{s}\right)=(0.5,1.6) \mathrm{km}, t_{0}=0 \mathrm{~s}$, and $v=2.4 \mathrm{~km} / \mathrm{s}$. When a cone is sliced vertically, a hyperbola is obtained. For this reason, for a set of receivers placed along a straight monitoring well (vertical or slanted), as depicted in Figure 1a, the time arrivals align along an apex-shifted hyperbola (Figure 1b). Should the receiver array not be aligned along a straight line, a fact that depends on the local conditions of the monitoring well and the engineering objectives, the arrival times will no longer align along a hyperbola. Thus, for the sake of generality, rather than assuming that the arrival times are aligned along a hyperbola, one may assume that in fact they are placed on a cone (or a hypercone in a 3D scenario). In practice, however, most monitoring wells are approximately straight along the extension of the receiver array; hence, the hyperbolic approximation is perfectly valid for constant velocity models. For nonconstant velocity models, raypaths are not straight lines. Thus, despite the fact that equation 1 can be generalized by redefining $r(x, z) / v$ as the traveltime from source to receiver, arrivals are no longer expected to be aligned 
along cones or hyperbolas. Nonetheless, the hyperbolic approximation is acceptable as long as the impact of the velocity heterogeneities on the observed arrival times is relatively small. For the sake of simplicity, the following analysis assumes a constant-velocity model. Later, we will consider the case in which the velocity is nonconstant.

The 3C microseismic synthetic data shown in Figure 2 correspond to an acquisition scheme similar to the one depicted in Figure 1a. Additive band-limited Gaussian noise was added with $\mathrm{S} / \mathrm{N}=10,3$, and 1 . The hyperbolic phase arrival observed in Figure $2 \mathrm{a}$ and $2 \mathrm{~b}$ corresponds to a $\mathrm{P}$-wave arrival for an eight-receiver vertical array. It is very easy to visually detect the phase arrival because its energy is much larger than the energy of the noise. Contrarily, in Figure $2 \mathrm{c}$, in which the $\mathrm{S} / \mathrm{N}$ is very low, the microseismic phase arrival can hardly be distinguished, even by a trained eye. Thus, to automatically detect a microseism, an analyst (or a computer algorithm) should be able to detect hyperbolic signal patterns in the presence of noise. For the sake of simplicity, it is generally assumed that the monitoring well is vertical (or approximately so for relatively short receiver arrays), and thus, the problem reduces to identifying an apex-shifted hyperbola in the $(z, t)$ domain (Velis et al., 2013; Sabbione et al., 2015).

\section{Pattern recognition}

Humans' (and animals') ability to visually recognize patterns is well known. This process, which is carried out almost effortlessly and instantaneously by the brain, is based on the visual perception of meaningful patterns and forms that "make sense" when a certain visual stimulus is received. Because patterns may contain a large amount of information, the perception of a given pattern may be a difficult task for a computer program, but not so for a living being such as primates that often use up to half of their total cortex space for processing visual stimuli (Felleman and van Essen, 1991). Usually, the process starts by assessing the evidence of a given number of features or a combination of features that characterize the input data and are associated with potential objects or parts of objects and their relationships. Then, the process involves the use of a large number of templates (or prototypes) to "match" the potential patterns or groups of patterns to the expected object (Galotti, 2014). These templates and prototypes constitute a priori information usually built based on past experiences and learning. How we interpret the visual stimuli in relation to this a priori information is what most differentiates humans (and animals) from machines or computer programs. Specifically, which algorithms or processes the brain actually uses to effortlessly detect objects remain poorly understood (DiCarlo et al., 2012).

In the context of microseismic event detection, the objective of a pattern recognition process is to devise a computer algorithm that is able to identify potential events from the observed data as a whole, just like a geophysicist analyst would do by visual inspection. Rather than considering every trace individually, a process that would include partial information only, whenever all traces are considered simultaneously, the human eye (or brain) immediately would associate coherent signals (patterns) to a given "object" (microseismic phase arrival). To this end, because microseism arrivals are expected to align along hyperbolic patterns, the proposed algorithm is devised to search for this kind of patterns within the input data. For the sake of efficiency, we use VFSA (Ingber, 1989) as a tool to maximize, with respect to the parameters that define all pos- sible hyperbolas that "make sense," an energy or coherence function computed within a hyperbolic time window that embraces any potential microseismic arrival (either the $\mathrm{P}$ - or $\mathrm{S}$-wave phase). This pattern recognition strategy will clearly ignore incoherent signals and any nonhyperbolic patterns. In addition, because VFSA requires the user to define a search range for all the parameters, nonrealistic hyperbolic patterns can be ignored too. Notice that, in practice, the selection of a search range does not represent any limiting assumption because they can be very large by virtue of the well-known searching efficiency of VFSA, as we will show in the "Results" section. Note that location and detection methods based on the grid search also rely on the selection of predefined search ranges, but their efficiency in terms of computational cost is much lower than VFSA (Lagos et al., 2014). Essentially, the method proposed by Sabbione et al. (2015) shares the same objective of searching for realistic hyperbolic patterns, but the calculations are computationally more intensive, especially when considering the hyperbolic Radon transform (Sabbione et al., 2013).

\section{Coherency and energy}

The pattern recognition problem can be solved by searching hyperbolic patterns within the given $3 \mathrm{C}$ data set. To this end, we need to define a measure of signal coherence or "energy" along all po-
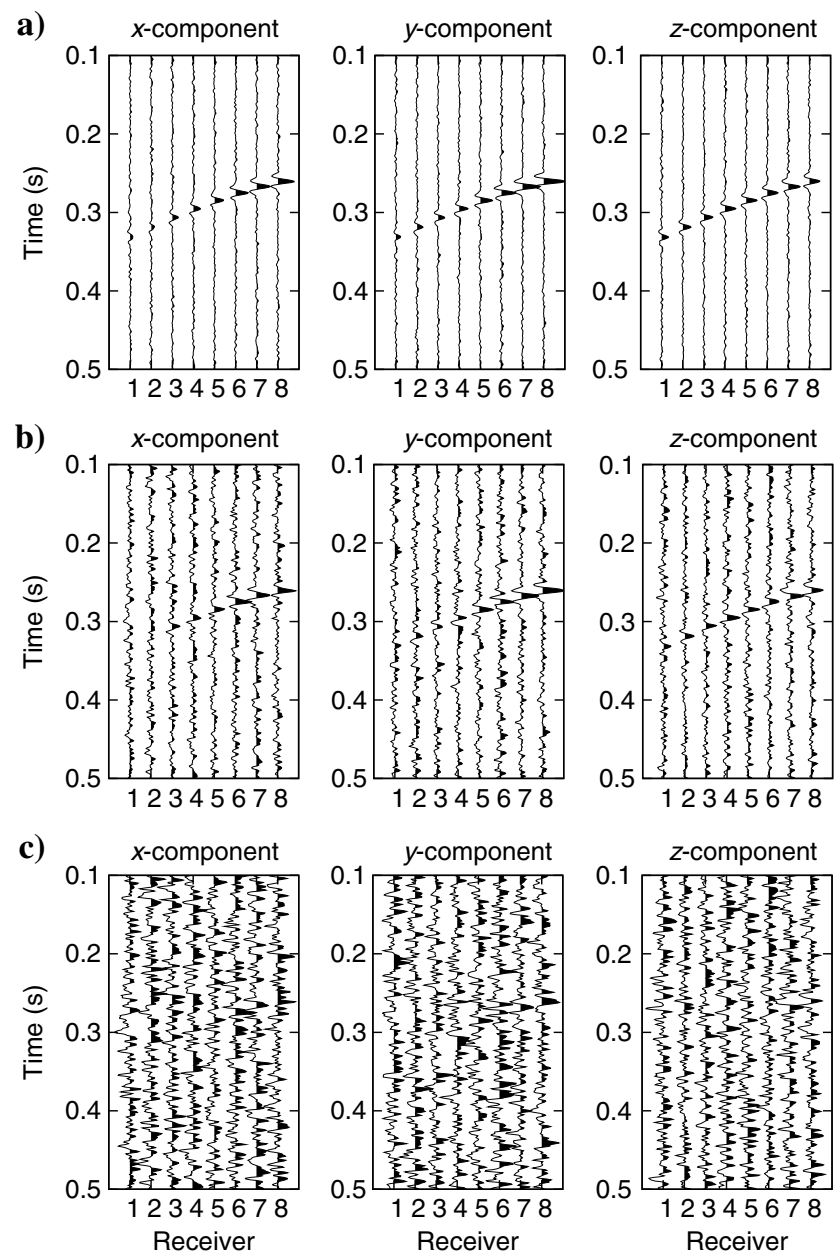

Figure 2. Synthetic data (a) $\mathrm{S} / \mathrm{N}=10$,

(b) $\mathrm{S} / \mathrm{N}=3$, and (c) $\mathrm{S} / \mathrm{N}=1$. 
tential hyperbolas (or cones), which are defined by $\left(x_{s}, z_{s}, t_{0}, v\right)$. Velis et al. (2013), for example, use the quantity

$$
G_{\mathrm{env}}\left(x_{s}, z_{s}, t_{0}, v\right)=\frac{1}{3 M} \sum_{i=1}^{M}\left(\hat{s}_{i}^{x}+\hat{s}_{i}^{y}+\hat{s}_{i}^{z}\right),
$$

where $\hat{s}_{i}^{c}$ is the $i$ th sample of the mean envelope $(c=x, y, z)$ along the trial hyperbola, and $M$ is the number of samples (width) of the search window. Clearly, when $G_{\text {env }}$ attains a maximum value, the probability of having a phase arrival is high. Note that this measure, which is similar to those used by Michaud and Leaney (2008), Gharti et al. (2010), and Sabbione et al. (2015), is particularly very sensitive to the energy of the phase arrival, irrespective of any phase changes.

An alternative measure is the average energy of the mean traces:

$$
G_{\mathrm{se}}\left(x_{s}, z_{s}, t_{0}, v\right)=\frac{1}{3 M} \sum_{i=1}^{M}\left[\left(\bar{s}_{i}^{x}\right)^{2}+\left(\bar{s}_{i}^{y}\right)^{2}+\left(\bar{s}_{i}^{z}\right)^{2}\right],
$$

where $\bar{s}_{i}^{c}$ is the $i$ th sample of the normalized mean trace $(c=x, y, z)$ along the trial hyperbola of width $M$. This energy measure, which we call stack energy for simplicity, is similar to the semblance used in
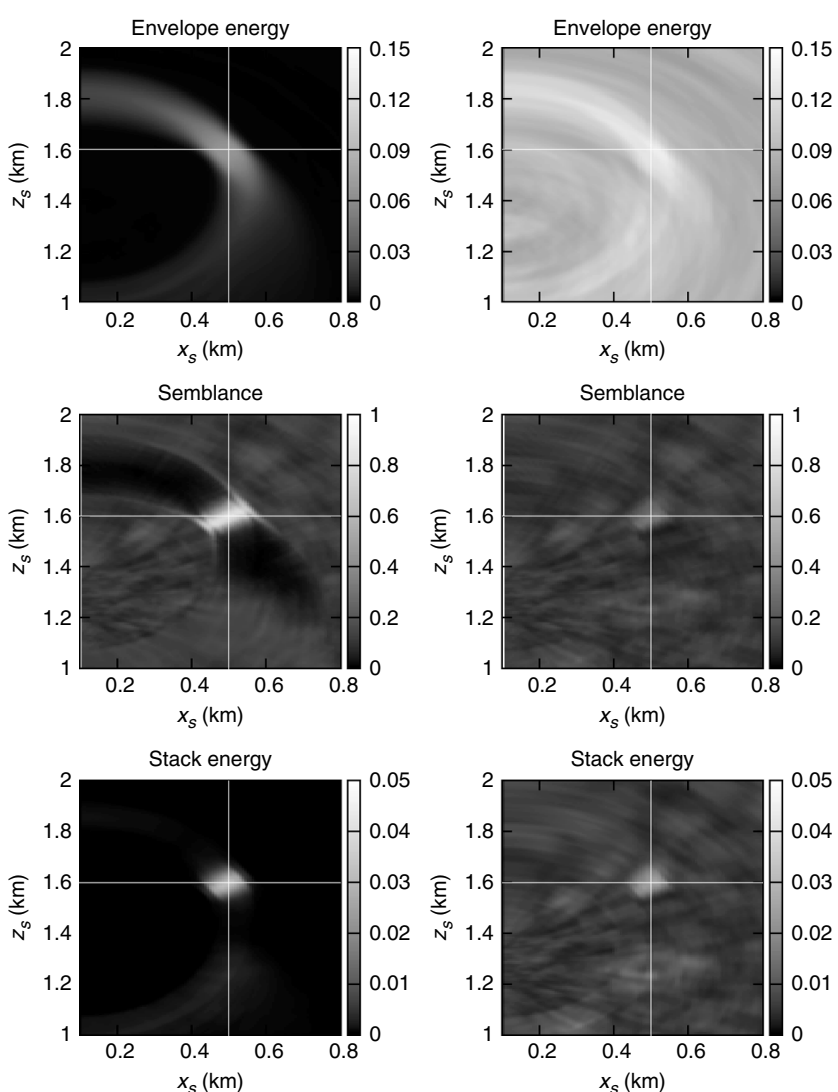

Figure 3. Envelope energy (top row), semblance (middle row), and stack energy (bottom row) associated with the microseismic data shown in Figure 2. In all cases, $t_{0}$ and $v$ have been fixed to their actual values. The source coordinates are denoted by the white crossing lines. Left column: data with $\mathrm{S} / \mathrm{N}=10$. Right column: data with $\mathrm{S} / \mathrm{N}=1$. conventional velocity analysis (Yilmaz, 2001) and reservoir characterization (Chopra and Marfurt, 2007). The conventional semblance, however, is related to an energy-normalized crosscorrelation, and it has a smaller dynamic range than unnormalized measures that represent estimates of signal energy (Neidell and Taner, 1971). Thus, semblance focuses on similarity rather than on energy, whereas the stack energy is sensitive to both.

Figure 3 (top row) shows $G_{\text {env }}\left(x_{s}, z_{s}, t_{0}, v\right)$ for fixed values $t_{0}=$ $0.0 \mathrm{~s}$ and $v=3.0 \mathrm{~km} / \mathrm{s}$ (the actual values used to generate the synthetics shown in Figure 2), and a search window of $0.03 \mathrm{~s}$. As expected, the global maximum is clearer in the case of a high $\mathrm{S} / \mathrm{N}$ (left) than in the case of a very low S/N (right). Similarly, Figure 3 (middle row) shows the conventional semblance. Note that although the global maximum can be distinguished more clearly in the high $\mathrm{S} / \mathrm{N}$ case (left) than in the low $\mathrm{S} / \mathrm{N}$ case (right), the semblance function is rather rough in both cases. Contrary to the envelope-based or the normalized semblance measures, which are devised to measure energy or similarity between waveforms, respectively, the stack energy is sensitive to the energy and similarity (Figure 3, bottom row). It is clear that the resolving power of the stack energy measure is the largest of the three measures, especially for a relatively high $\mathrm{S} / \mathrm{N}$. Naturally, other energy or coherency measures with high resolving
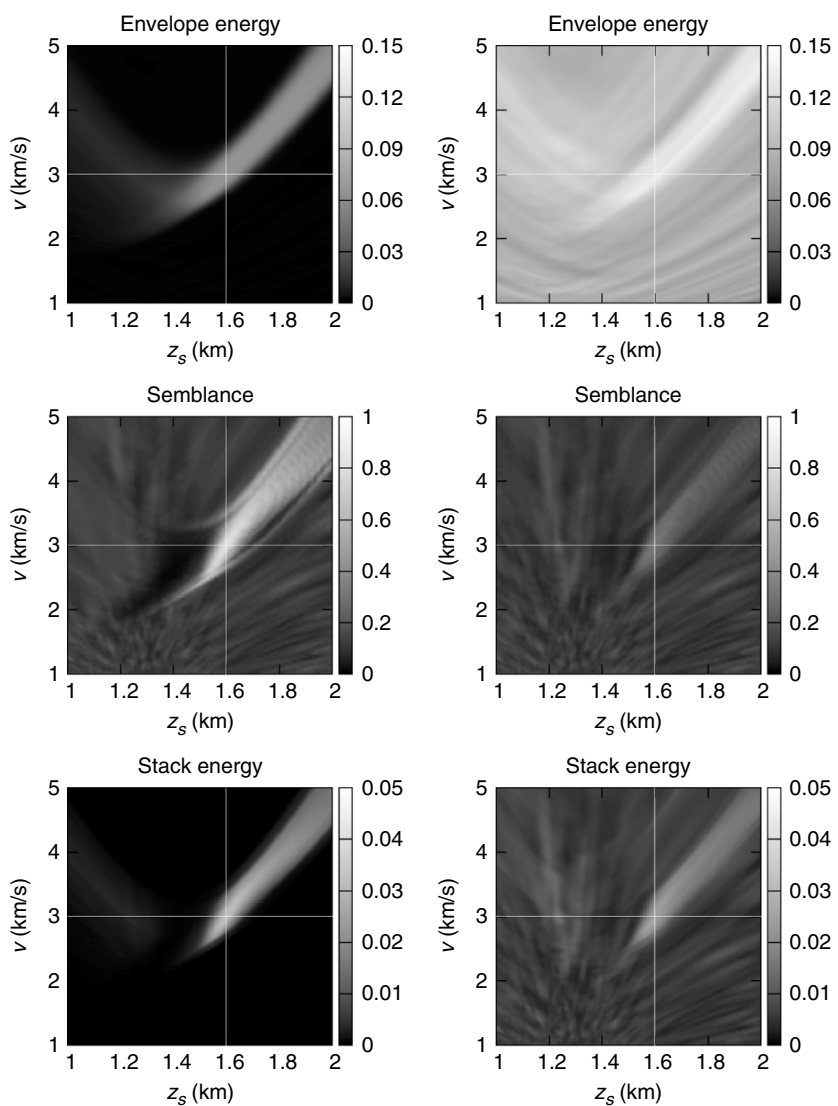

Figure 4. Envelope energy (top row), semblance (middle row), and stack energy (bottom row) associated with the microseismic data shown in Figure 2. In all cases, $t_{0}$ and $x_{s}$ have been fixed to their actual values. The source $z_{s}$ coordinate and actual velocity are denoted by the white crossing lines. Left column: data with $S / N=10$. Right column: data with $\mathrm{S} / \mathrm{N}=1$. 
power could be taken into account, such as those proposed by Sacchi (1998) and Ursin et al. (2014) in the context of velocity analysis.

Similar behavior is observed when $t_{0}$ and $x_{s}$ are fixed and $z_{s}$ and $v$ are varied (Figure 4). For the analyzed data set, the stack energy provides the highest resolving power of the three tested measures, the semblance has a lower resolving power and is quite rough, and the envelope energy has the lowest resolving power but is the smoothest. Nonetheless, as compared with Figure 3, the global maximum is much less pronounced in all cases. This occurs because there exist several hyperbolas that embrace the microseismic phase arrival due to the short aperture of the array (see Figure 2). This nonuniqueness problem does not represent any serious issue for detecting the microseismic phase arrival, as shown in the "Results" section. In contrast, it may severely affect the location problem, for the accurate position of the source in the time-space domain is poorly constrained when a single monitoring well is used.

In summary of the three tested measures, the envelope energy is the smoothest and exhibits the lowest resolving power, which should not complicate the detection problem. However, the stack energy shows the highest resolving power, but the global maximum might be harder to find because it occupies a smaller portion of the model space. Finally, the semblance has a considerable resolving power, but it is the roughest and the most sensitive to noise. Hence, the use of envelope-based measures might be preferable for solving the detection problem. This preliminary conclusion will be supported by the results shown in next sections. To solve the location problem (not addressed in this study), a measure with higher resolving power and relatively robust to random noise, such as the stack energy, might be desirable.

\section{Detection}

Once we select an appropriate coherency-energy measure, which we call $G$ for the sake of generality, the next step is to find the parameters that lead to their maximum and associate them to a potential microseismic phase arrival. In practice, we minimize the objective function,

$$
\Phi\left(x_{s}, z_{s}, t_{0}, v\right)=1-G\left(x_{s}, z_{s}, t_{0}, v\right)
$$

provided the data have been previously normalized so that $0 \leq G \leq 1$.

Thus, the pattern recognition problem becomes a multidimensional optimization problem. One of the most effective methods to find the global minimum of a function is perhaps the grid search, provided that the parameter discretization is fine enough (Lagos et al., 2014). In the 2D case illustrated in Figure 1, in which the $x$-axis is aligned along the azimuth (which is assumed to be known a priori), $\Phi\left(x_{s}, z_{s}, t_{0}, v\right)$ depends on four parameters. Thus, a grid search might be very time consuming and not very practical when processing large data sets. A gradient-based method is not recommended either because the roughness of $\Phi$ may lead to local minima. Alternatively, we use a very efficient global search technique such as VFSA (Ingber, 1989), a powerful optimization algorithm that converges much faster than conventional SA approaches. Furthermore, because the unknown model variables are allowed to vary continuously, the accuracy of the results, and the number of iterations required to reach the global minimum, do not depend on the parameter discretization.
It is important to remark that obtaining the global minimum of $\Phi$ does not guarantee that a microseism phase arrival has been detected because the analyzed time window may contain none. Thus, a criterion is needed to assess the presence of an event or a "false positive." To this end, we propose to compute the following energy ratio as an indicator of the presence of a phase arrival:

$$
R_{E}=E / \bar{E}_{n}
$$

where $E$ is the energy of the potential phase arrival and $\bar{E}_{n}$ is the corresponding mean energy of the noise, which can be estimated by averaging the energy of a certain number of random hyperbolas. Clearly, when $R_{E}$ is close to 1 , the probability of having a phase arrival is low, and the detection should be discarded or checked visually by the analyst. However, when $R_{E}$ is much larger than 1 , the probability of having a phase arrival is high.

\section{Denoising}

Denoising is carried out after a phase arrival has been detected. For this purpose, we extract the detected hyperbolic window to focus on the microseismic phase arrival. Then, the data within the search window of length $M$ that contains a phase arrival are viewed as a rectangular matrix $S$ of size $M \times N$, in which $N$ is the number of traces. The SVD of $S$ is given by

$$
S=U \Sigma V^{T}
$$

where $U$ is a $M \times N$ column-orthogonal matrix, $\Sigma$ is an $N \times N$ diagonal matrix with positive or zero elements called singular values, and $V^{T}$ is the transpose of an $N \times N$ column-orthogonal matrix (Press et al., 1992). This matrix decomposition always exists, and it is unique. The columns of $U$ (left singular vectors of $S$ ) are the eigenvectors of the matrix $S S^{T}$, whereas the columns of $V$ (right singular vectors of $S$ ) are the eigenvectors of the matrix $S^{T} S$. In general, the eigenvectors are ordered so that their corresponding singular values $\sigma_{i}$, which are equal to the square roots of the corresponding eigenvalues, satisfy $\sigma_{1} \geq \sigma_{2} \geq \cdots \geq \sigma_{N}$. Then, $S$ can be expressed as

$$
S=\sum_{i=1}^{q} \sigma_{i} \mathbf{u}_{i} \mathbf{v}_{i}^{T},
$$

with $q=N$, and $\mathbf{u}_{i}$, and $\mathbf{v}_{i}$ being the corresponding eigenvectors. Note that $\mathbf{u}_{i} \mathbf{v}_{i}^{T}$ constitute basis images of rank one that form an orthogonal basis for image representation (eigenimages). Thus, if $q<N$, the sum 9 leads to an approximate matrix $S_{q}$ of rank $q$. In addition, $S_{q}$ is such that the Frobenius norm of the difference between $S$ and $S_{q}$ is minimized, and the norm of each eigenimage is equal to the corresponding singular value (Golub and Van Loan, 1989).

In many applications, such as image compression (Gonzalez and Wood, 2007), usually a large number of basis images can be neglected. This is especially the case of a microseismic arrival contained in the matrix $S$ because all the signals are expected to share similar waveforms, at least for relatively short arrays and observational distances. Indeed, in the noise-free case, if all the $N$ signal arrivals were equal to within a scale factor, then $\sigma_{i}=0$ for $i=2, \ldots, N$, and $S$ would be a rank one matrix. Thus, $S_{1}=\sigma_{1} \mathbf{u}_{1} \mathbf{v}_{1}^{T}$ would contain all the signal energy. As a result, all waveforms will be scaled versions of each other and most random noise and incoherent signals would be 
attenuated, as we will show in the "Results" section. When waveforms vary, the level of noise attenuation and waveform "homogenization" can be controlled by the number of singular values used in the approximation because the signal energy would be contained in the first (and largest) eigenimages.

\section{Synchronization}

Until now, we have assumed that the underlying velocity model was constant. This assumption has allowed us to describe the microseismic time arrivals by cones and/or hyperbolas. Clearly, when the velocity model is heterogeneous, the signals are not expected to align in such a geometrically simple way and a single parameter $v$ used to fit the arrivals as in equation 3 is questionable. In spite of this, as shown by Blias and Grechka (2013), a homogeneous medium with an effective constant velocity may provide suitable constraints to solve the microseismic event location problem and constitute a simple and effective strategy to fit the arrival times acceptably well in most scenarios. Naturally, the accuracy of the estimated hypocenters degrades with velocity heterogeneity. In solving the detection problem, however, the constant velocity assumption is less restrictive because the velocity is only used in defining the search window. In this sense, it is enough that it contains the microseismic phase arrival, but it is not necessary that it aligns very accurately along the actual hyperbolic phase arrival. It is emphasized that, as pointed out by Blias and Grechka (2013), this effective velocity represents a fitting parameter in the same way as the stacking velocity is a fitting parameter in conventional velocity analysis of seismic reflection data. Therefore, an estimate of the actual velocity model is not required for it will be obtained as a by-product when equation 6 is minimized.

Nevertheless, the proposed method is general enough so as to accommodate heterogeneous models whenever these models are available a priori. In fact, the energy or coherence measure is computed along a time-varying window, which may or may not follow a simple geometric curve or surface, depending on the velocity used in equation 1 . For nonconstant velocities, arrival time $t$, which defines the time-varying window, can be computed using either ray tracing or any other technique for every tested triplet $\left(x_{s}, z_{s}, t_{0}\right)$, instead of $\left(x_{s}, z_{s}, t_{0}, v\right)$, at each SA iteration. Thus, although it is computationally more demanding, the number of unknowns is reduced by one and the optimization of the corresponding cost function should not be a problem provided the input velocity model is accurate enough. Actually, most event location methods require the user to provide an adequate velocity model. This velocity model, which is usually derived from sonic logs, should be properly calibrated before proceeding to estimate the hypocenters (Maxwell, 2014).

Here, we provide an alternative strategy to allow for heterogeneous velocity models when these are not available. The usefulness of this strategy relies on the assumption that the homogeneous velocity approximation is good enough so as to provide a hyperbola that embraces the potential phase arrival, whether the individual arrivals at each receiver are aligned or not. Then, the individual detected arrivals are synchronized by adding appropriate time shifts. By this means, it is possible to account for possible deviations between the modeled and observed arrival times due to the velocity model assumption. This is a very important process for the denoising stage because SVD filtering assumes that the arrivals are aligned along the final search window. Thus, once the phase arrival has been detected in the framework of the constant velocity model assumption, we proceed to compute the average trace envelope within the final search window and time shift every individual arrival so as to maximize the crosscorrelation between the average envelope and the corresponding individual envelopes. This simple crosscorrelation-based synchronization process guarantees that all arrivals are properly aligned before carrying out the denoising using the SVD. Finally, the time-shift corrections are undone to obtain the corresponding denoised microseismic phase arrival. The described strategy is specially suited for relatively short arrays and when the impact of the velocity heterogeneities on the time shifts are relatively small. Nevertheless, the strategy can also be applied when the velocity model is available. In this case, the synchronization process could account for possible deviations between the modeled and observed phase-arrival times due to the uncertainties in the velocity model.

\section{Deflation}

Another assumption that we have used so far is that there is a single phase arrival within the analyzed time window. However, data may contain $\mathrm{P}$ - and $\mathrm{S}$-wave arrivals associated with one or more microseismic events. Rather than detecting and denoising all signal arrivals simultaneously, we propose to carry out the detection and denoising of each individual signal arrival separately by means of a deflation procedure. In this sense, once a phase arrival has been detected and denoised using the described methods, the resulting data are subtracted from the original data, leading to a residual data set containing any other phase arrival that may have not been detected, denoised, and removed yet. Then, the residual is treated as a new data set for the detection and denoising. This deflation process is repeated until no new phase arrivals are detected (e.g., until $R_{E}$ is smaller than a given threshold value). Note that because the detection process involves the minimization of the cost function 6 by means of SA, which is equivalent to locate the most energetic and/or coherent hyperbolic phase arrival within the analyzed time window, the deflation procedure implies that the most significant phase arrivals will be detected first. At the end of the deflation process, the residual is expected to contain noise only.

The described deflation process resembles the well-known matching pursuit algorithm, in which seismic traces are decomposed into a series of "atoms" of decreasing energy selected from a large and redundant dictionary of basis functions (Mallat and Zhang, 1993). Usually, dictionaries are built based on adaptive time-frequency wavelets (e.g., Gabor or Morlet wavelets) so that the time-varying signal structure can be correctly represented by a relatively small number of atoms (Liu and Marfurt, 2005; Wang, 2010). In the proposed deflation process, instead of using predefined basis functions or wavelets, the microseismic signals are represented by a reducedrank approximation of the SVD of the detected phase arrivals along hyperbolic windows.

\section{RESULTS}

\section{Synthetic example 1}

First, we test the method using the $3 \mathrm{C}$ microseismic synthetic data shown in Figure 2. These data sets contain a single microseismic phase arrival and correspond to a constant velocity model. Thus, cost function 6 is dominated by a single global minimum 
(although small local minima exist, as shown in Figures 3 and 4), and the deflation procedure will not be required. No synchronization would be required either because the individual arrivals are aligned along hyperbolas. The deflation and synchronization procedures will be illustrated in synthetic example 2. For statistical purposes, and for the detection process only, in each case we perform 100 realizations and averaged the results. The time window width involved in the calculation of $G$ was $30 \mathrm{~ms}$, the maximum number of SA iterations was 1000, and the search ranges were set as follows: $x_{s}=0-1 \mathrm{~km}$, $z_{s}=0-2 \mathrm{~km}, t_{0}=0-0.2 \mathrm{~s}$, and $v=1-5 \mathrm{~km} / \mathrm{s}$.

Table 1 summarizes the average results and the corresponding standard deviations. The phase arrival was detected in all the cases with a high degree of confidence, as denoted by the fact that $R_{E}>>1$ except for the $\mathrm{S} / \mathrm{N}=1$ case where $R_{E}=1.39 \pm 0.1$. We observe that the uncertainties of the various parameters increase with a decreasing $\mathrm{S} / \mathrm{N}$, as expected, except for $x_{s}, t_{0}$, and $\Delta t_{\mathrm{rms}}$ for $\mathrm{S} / \mathrm{N}=1$, and $R_{E}$ in all cases. The uncertainties associated with the hyperbola parameters are all very large, even for $\mathrm{S} / \mathrm{N}=10$, and their variations with $\mathrm{S} / \mathrm{N}$ are not very significant. Contrarily, the uncertainties associated with $G$ and the root-mean-square (rms) error between the actual arrival times $t\left(x_{i}, z_{i}\right)$ and the calculated arrival times $\hat{t}\left(x_{i}, z_{i}\right)$ are relatively low. The arrival time rms error is computed using

$$
\Delta t_{\mathrm{rms}}=\left\{\frac{1}{N} \sum_{i=1}^{N}\left[t\left(x_{i}, z_{i}\right)-\hat{t}\left(x_{i}, z_{i}\right)\right]^{2}\right\}^{1 / 2} .
$$

As for the denoising stage, the results are shown in Figure 5 (left column) for a particular realization of those averaged in Table 1. In each case, we use a single eigenimage to approximate the data within the final search window. We observe that the waveforms and amplitudes were recovered very well, especially for the higher $\mathrm{S} / \mathrm{N}$ cases (Figure 5a and 5b, left column). In these cases, the normalized crosscorrelations between the noise-free data sets and the corresponding denoised data sets are very high (greater than 0.95), as shown in Table 2. Although less accurately, the microseismic signal was also recovered in the very low $\mathrm{S} / \mathrm{N}$ case (Figure $5 \mathrm{c}$, left column). Overall, these results are quite reasonable, considering the fact that the actual signal is badly contaminated in the $\mathrm{S} / \mathrm{N}=3$ case (Figure 5b, left column), and they are almost undistinguished (if not hidden at all) in the $\mathrm{S} / \mathrm{N}=1$ case (Figure 5c, left column).

For comparison, Figure 5 (right column) shows the results of the detection and denoising using the apex-shifted hyperbolic Radon transform approach described in Sabbione et al. (2013). For the two higher $\mathrm{S} / \mathrm{N}$ cases, we observe that the accuracy of the results is very similar to the accuracy obtained using the proposed SVD filtering, yielding very high crosscorrelation values (see Table 2). However, the Radon approach degrades significantly in the very low $\mathrm{S} / \mathrm{N}$ case. Note that for a fair comparison, we calculate the crosscorrelations using the denoised images muted with the same hyperbolic window used in the SVD filtering.

Figure 6 shows the convergence curves for the $\mathrm{S} / \mathrm{N}=3$ case (synthetic example 1), using the envelope energy $G_{\text {env }}$ (left panel) and the stack energy $G_{\mathrm{se}}$ (right panel), respectively. Note that in most cases, the convergence is achieved well before 1000 iterations, especially when $G=G_{\text {env }}$. The convergence is faster when using the envelope energy measure because it is smoother (and easier to minimize/maximize) than the stack energy measure (see Figures 3 and 4). The same behavior is observed for the cases with $\mathrm{S} / \mathrm{N}=10$ and 1 (not shown). In terms of computational cost, the final solution for a single SA run using either measure is obtained almost instantly on a standard PC, making the method suitable for real-time processing.

When we compare the arrival times plotted in Figure 7, we notice that, due to its higher resolving power, the greater accuracy is obtained when using the stack energy (dotted line) than using the envelope energy (dashed line) in the $\mathrm{S} / \mathrm{N}=1$ case (Figure 7 , right panel). However, when the noise level is moderate to low $(\mathrm{S} / \mathrm{N}=10$, left panel of the same figure), both measures exhibit similar accuracy, in spite of a relatively small bias of a few milliseconds. We want to stress that for the sake of the phase-arrival detection process, both measures behave satisfactorily well, even for the $\mathrm{S} / \mathrm{N}=1$ case, in which the signal is almost indistinguishable. Nonetheless, for the SVD filtering, it is clear that we need to obtain a hyperbolic window that follows as closely as possible the curvature of the actual phase arrival. A moderate bias is of no concern because the individual waveforms would add up in phase anyway. On the contrary, any deviation from the actual curvature, as shown in the right panel of Figure 7 for the envelope-energy case, would certainly diminish the quality of the results.

Quantitatively, the accuracy of the results was already assessed by analyzing the normalized crosscorrelations between the clean (noise free) data and the denoised data as shown in Table 2. Because the clean data are not available when dealing with field data sets, the accuracy of the results can be assessed by analyzing, instead, the normalized crosscorrelations between the raw (noisy) and the denoised data. Theses quantities are depicted in Figure 8 (left column), on a channel-by-channel basis. Note that crosscorrelation values greater than $0.7-0.8$ are obtained for most microseismic arrivals, except for the very low S/N case. Crosscorrelation between the actual and the denoised signals is viewed as an $\mathrm{S} / \mathrm{N}$ indicator that can be used for subsequent microseismic data analysis processes. For example, they could be used as weights when averaging the individual azimuths derived from a hodogram analysis.

\section{Synthetic example 2}

The purpose of the second synthetic example is to test the synchronization and deflation processes. This example illustrates the case in which the underlying velocity model is not constant and there is more than a single phase arrival. Essentially, we use the same source-receiver geometry as in the case of the data shown

Table 1. Mean and standard deviation after 100 realizations $\left(k_{\max }=1000, G=G_{\mathrm{se}}\right)$. Actual event parameters are $\left(x_{s}, z_{s}, t_{0}, v\right)=(0.5,1.6,0.0,3.0)$.

\begin{tabular}{llll} 
& \multicolumn{1}{c}{$\mathrm{S} / \mathrm{N}=10$} & \multicolumn{1}{c}{$\mathrm{S} / \mathrm{N}=3$} & \multicolumn{1}{c}{$\mathrm{S} / \mathrm{N}=1$} \\
\hline$x_{s}(\mathrm{~km})$ & $0.534 \pm 0.122$ & $0.531 \pm 0.126$ & $0.467 \pm 0.110$ \\
$z_{s}(\mathrm{~km})$ & $1.648 \pm 0.086$ & $1.640 \pm 0.092$ & $1.680 \pm 0.161$ \\
$t_{0}(\mathrm{~s})$ & $0.002 \pm 0.054$ & $0.000 \pm 0.057$ & $-0.003 \pm 0.050$ \\
$v(\mathrm{~km} / \mathrm{s})$ & $3.139 \pm 0.411$ & $3.068 \pm 0.420$ & $2.901 \pm 0.568$ \\
$\Delta t_{\text {rms }}(\mathrm{s})$ & $0.0031 \pm 0.0016$ & $0.0039 \pm 0.0021$ & $0.0044 \pm 0.0009$ \\
$G$ & $0.0698 \pm 0.0007$ & $0.0079 \pm 0.0008$ & $0.1050 \pm 0.0011$ \\
$R_{E}$ & $9.17 \pm 1.67$ & $3.67 \pm 0.29$ & $1.39 \pm 0.10$ \\
\hline
\end{tabular}


in Figure 2, but we include the S-wave arrival with $v_{s}=2.4 \mathrm{~km} / \mathrm{s}$. For the optimization process, we use the same parameters as in the example 1: a time window width of $30 \mathrm{~ms}$, a maximum of $1000 \mathrm{SA}$ iterations, and the following search ranges: $x_{s}=0-1 \mathrm{~km}$, $z_{s}=1-2 \mathrm{~km}, t_{0}=0-0.2 \mathrm{~s}$, and $v=1-5 \mathrm{~km} / \mathrm{s}$. To simulate possible deviations between the modeled and observed arrival times due to velocity model inaccuracies, as discussed in the "Synchro- nization" section, we add to each individual arrival time random perturbations drawn from a uniform distribution in the range $(-10,10) \mathrm{ms}$. A portion of the resulting data $(\mathrm{S} / \mathrm{N}=10)$ is shown in Figure 9 (top row). Notice that the actual $\mathrm{S} / \mathrm{N}$ is much lower than 10 for the $\mathrm{P}$-wave in general and for the $y$-component of the $\mathrm{S}$-wave in particular. In addition, the P-wave arrivals corresponding to the first 3 and 4 receivers exhibit a very low S/N. The same observation a)
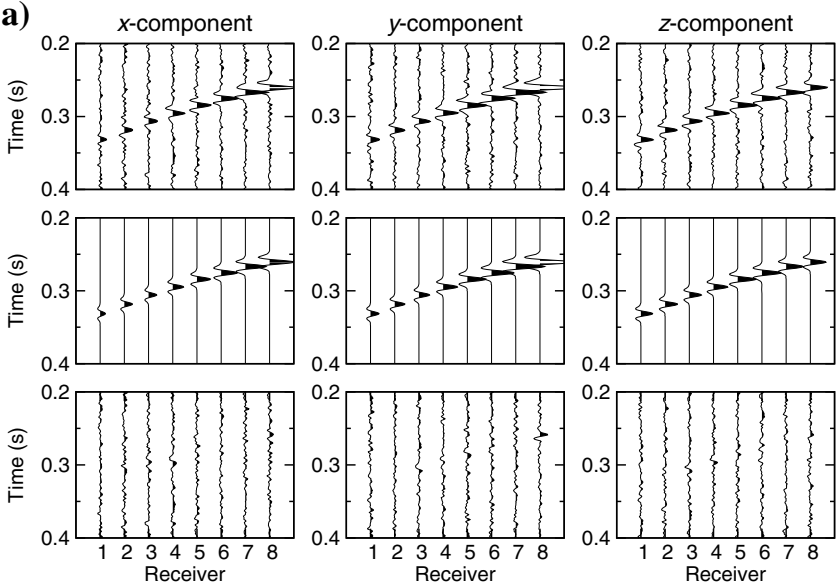

b)
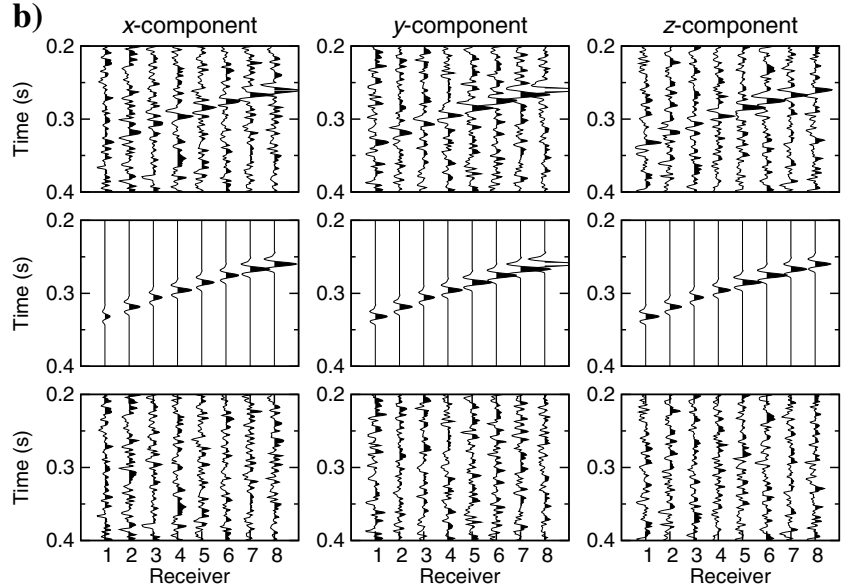

c)
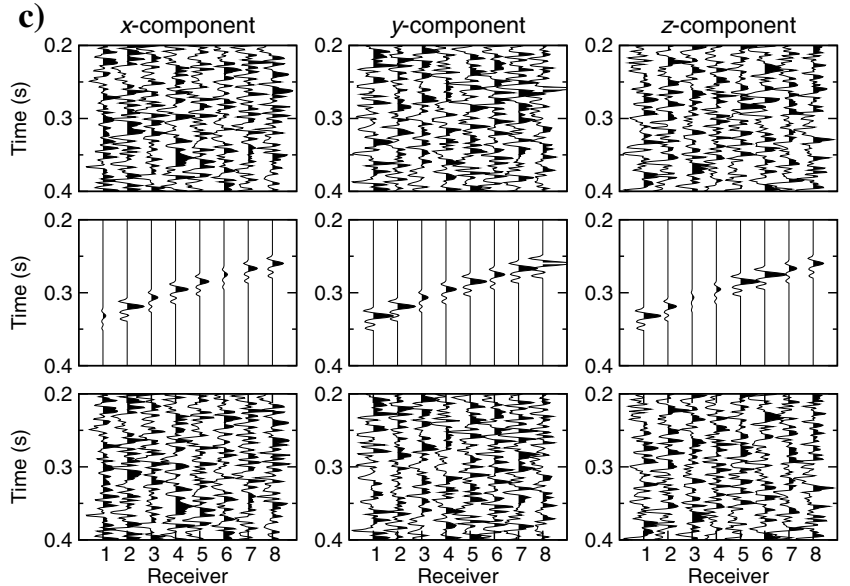
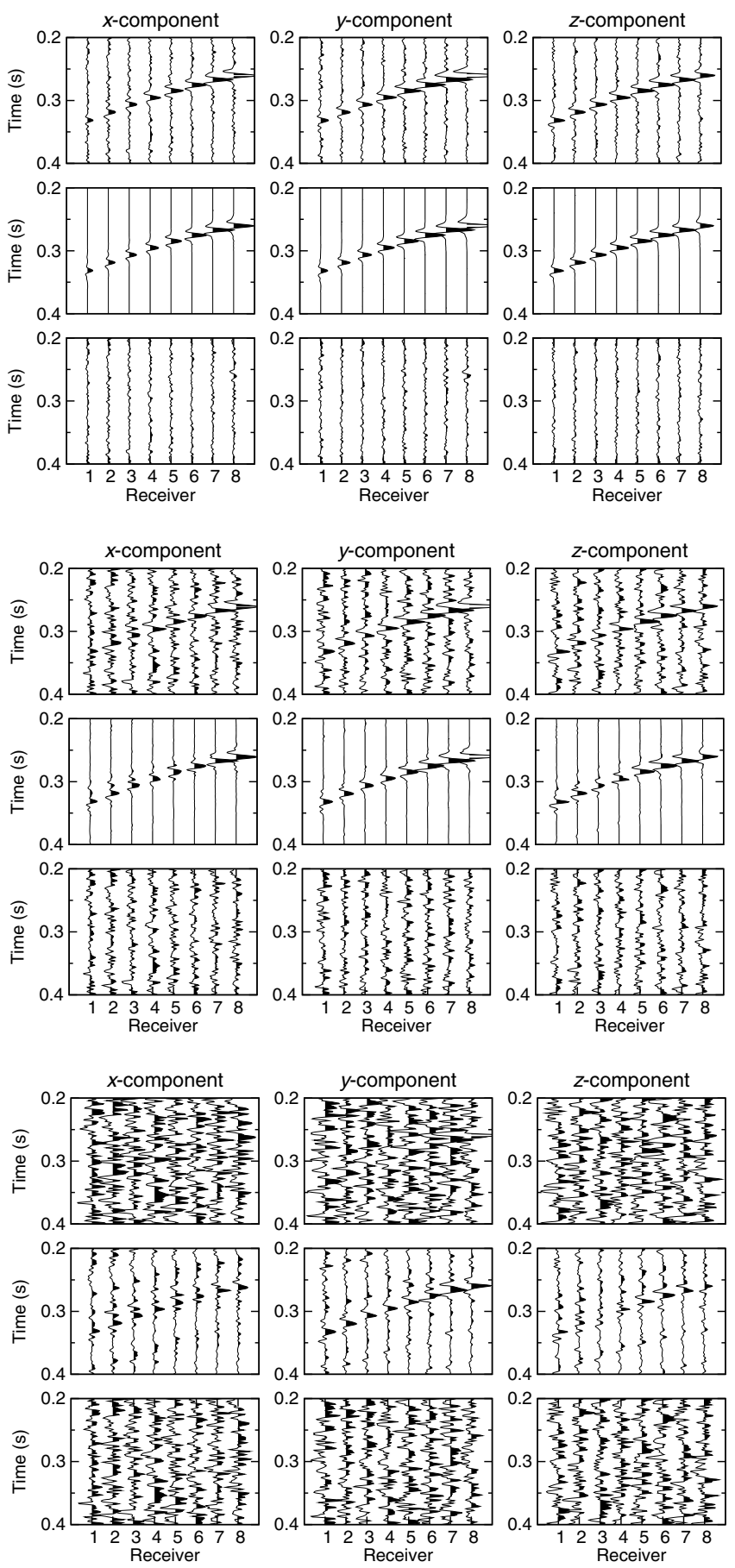

Figure 5. Microseismic signal denoising (synthetic example 1) using the proposed method (left column) and the hyperbolic Radon transform approach (right column): (a) $\mathrm{S} / \mathrm{N}=10$, (b) $\mathrm{S} / \mathrm{N}=3$, and (c) $\mathrm{S} / \mathrm{N}=1$. In each case, rows 1 to 3 represent the actual data, the detected/ denoised phase arrival, and the residual, respectively. 
is valid for receivers $5-8$ of the $S$-wave arrivals that include a polarity reversal due to the associated source radiation pattern. Figure 10 shows the corresponding arrival times (square dots) and the unperturbed hyperbola (solid line).

Because we have two phase arrivals, the deflation process for this particular example consists of applying the described detection and denoising procedures twice. The synchronization step was used to align the individual arrivals before denoising via the SVD filtering. In the first iteration of the deflation procedure, the most significant phase arrival (the S-wave arrival) was detected and denoised (Figure 9, second row). The corresponding residual (Figure 9, third row) was then input to the detection/denoising process again, leading to the results shown in Figure 9, fourth row. The fifth row of the same figure shows the corresponding residual. Finally, the bottom row shows the full data reconstruction, which is the result of adding up the data in rows two and four. We observe that the P- and S-wave phase arrivals were recovered very accurately despite the fact that the individual arrivals are not aligned along a perfect hyperbola. The resulting hyperbolas estimated after the minimization of cost function 6 are depicted in Figure 10 (dashed lines), together with the arrival times derived from the crosscorrelation-based synchronization (cross dots).

\section{Field data example}

We select three field records that were acquired in a monitor well located $500 \mathrm{~m}$ away from the extraction well using a vertical array of eight triaxial receivers spaced $30 \mathrm{~m}$. Figure 11 shows a halfsecond window that contains a microseismic phase arrival and the final window after automatic detection. The search window length was set to $20 \mathrm{~ms}$, the maximum number of iterations was set to 1000 , and the search ranges were set as follows: $x_{s}=0-1 \mathrm{~km}, z_{s}=0-4 \mathrm{~km}, t_{0}=0-0.2 \mathrm{~s}$, and $v=1-5 \mathrm{~km} / \mathrm{s}$. In all cases, the phase arrival was detected accurately despite the fact that it is almost missing in some of the components (e.g., the $x$-component), probably due to the radiation pattern, coupling effects, or noise. Furthermore, some channels do not exhibit any useful information either because there is some problem with the receiver (e.g., channel 5 in the $x$-component) or because of the very low $\mathrm{S} / \mathrm{N}$ (e.g., the $z$-component channels in the poor and very poor quality data records).

Figure 12 (left column) shows the resulting denoised data sets ( $q=3$ in equation 9). First, we observe that the phase arrival was accurately detected in all the cases, even when the $\mathrm{S} / \mathrm{N}$ is very low (Figure $12 \mathrm{c}$, left column). The corresponding confidence indicators $R_{E}$ were 6.2, 2.5 , and 1.9 for the moderate, poor, and very poor quality data sets, respectively. The synchronization process, although applied, did not yield any significant time shifts. The deflation process, however, led to $R_{E}$ values of $1.3,1.2$, and 1.3 , for the moderate, poor, and very poor quality data records, respectively. These "close-to-one" values indicate that the probability of having a second microseismic arrival or wave phase within the analyzed time window is very low, a fact that can be corroborated by visually inspecting the original data as shown in Figure 11.

In Figure 12 (left column), we also observe that consistent waveforms were recovered reasonably well in most channels, especially in the moderate quality data case and at those channels of the poor and very poor quality data sets in which the $\mathrm{S} / \mathrm{N}$ is not that severe. For those channels in which the $\mathrm{S} / \mathrm{N}$ is extremely low (e.g., channels 1 and 2 on most cases), the denoised data show waveforms that are compatible with the signal energy and the nearby waveforms. Finally, we note that the results of the denoising using the Radon approach (Figure 12, right column) are comparable to those obtained using SVD filtering, except for the very poor quality data case.

Finally, Figure 8 (right column) shows the corresponding crosscorrelations between the raw data and the denoised data. As expected, many channels exhibit very low crosscorrelation values, a fact that is interpreted as an indicator that the information contained in the resulting denoised arrivals may not be very reliable. This is the case, for example, for channels 6 and 8 of the $x$-component in the very poor quality data set, in which the crosscorrelations are approximately 0.1 and lower. On the contrary, some channels and/or components exhibit relatively high crosscorrelation values ( 0.7 and above), suggesting that the corresponding wave arrivals can be used with higher confi-

Table 2. Normalized crosscorrelation between the noise-free data sets and the corresponding denoised data sets using the SVD filtering and the Radon approach (synthetic example 1).

\begin{tabular}{ccccccc} 
& \multicolumn{2}{c}{$\mathrm{S} / \mathrm{N}=10$} & \multicolumn{2}{c}{$\mathrm{S} / \mathrm{N}=3$} & \multicolumn{2}{c}{$\mathrm{S} / \mathrm{N}=1$} \\
\cline { 2 - 7 } & SVD & Radon & SVD & Radon & SVD & Radon \\
\hline$x$ & 0.987 & 0.986 & 0.968 & 0.920 & 0.748 & 0.572 \\
$y$ & 0.987 & 0.989 & 0.978 & 0.963 & 0.838 & 0.818 \\
$z$ & 0.984 & 0.991 & 0.970 & 0.933 & 0.799 & 0.724 \\
\hline
\end{tabular}

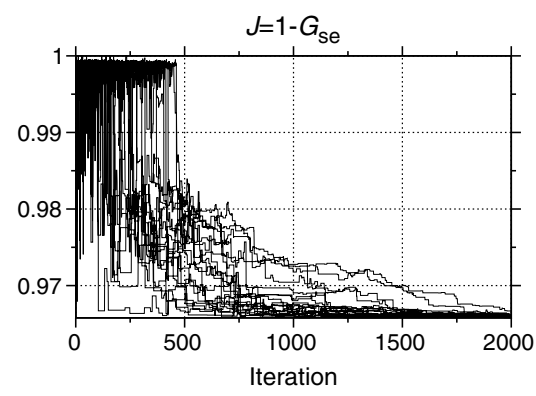

(20 realizations) for the Figure 6 . Very fast simulated annealn
synthetic example 1 with $S / N=3$.
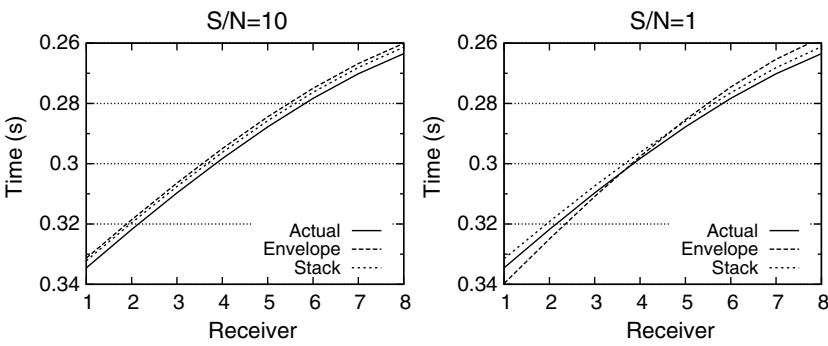

Figure 7. Average arrival times (100 realizations) for the synthetic example 1 when using the envelope energy and the stack energy measures. 
dence for further data processing and/or analysis of the microseismic data.

\section{DISCUSSION}

The statistical results summarized in Table 1 for synthetic example 1 show that the estimated source parameters (i.e., the cone apex) for the three tested noise levels are relatively uncertain, but the arrival times are very accurate. Moreover, the variations in the standard deviations do not show a clear increase with decreasing $\mathrm{S} / \mathrm{N}$. This suggests that the optimization process (and the detection of the phase arrival) is robust to random noise and accurate, despite the fact that there are many similar hyperbolic windows that include the microseismic signal. For this reason, the estimated hyperbola parameters should not be assumed to correspond to the actual location and occurrence time of the source, but rough estimates of the actual source parameters. In contrast, the resulting arrival times are very accurate estimates of the actual values, implying that the proposed technique may be used as an automatic picking tool.

Contrarily to solving the detection problem, the location problem that aims to determine the source coordinates (cone or hypercone apex) is a much harder task because the observed arrival times sample a small portion of the cone or hypercone as shown in Figure 1a. Furthermore, the underlying velocity model is usually uncertain. Thus, the accurate position of the cone or hypercone apex in the time-space domain is poorly constrained when a single monitoring
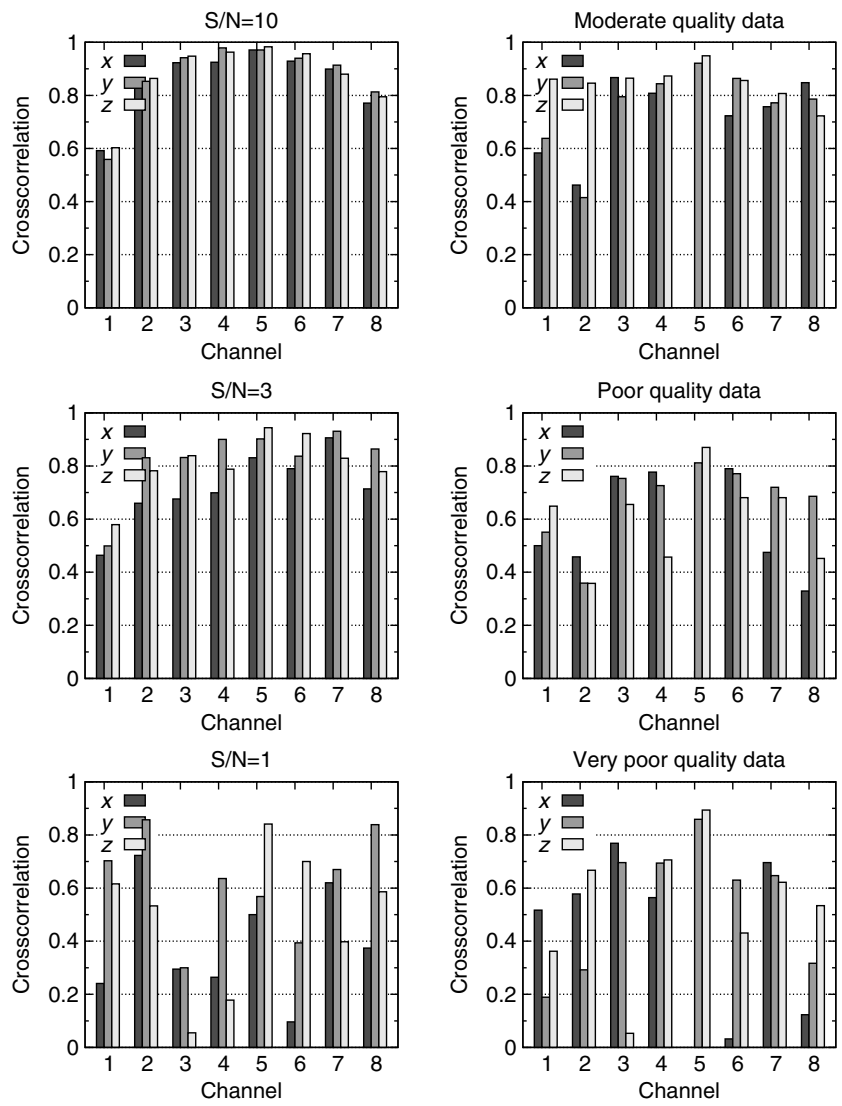

Figure 8. Normalized crosscorrelations between the individual raw and denoised signals for the synthetic example 1 (left column) and the field data example (right column). well is used, and further constraints are required to reduce the uncertainty in most field experiments (e.g., by adding a second monitoring well). Hence, for $2 \mathrm{D}$ scenarios (in which the azimuth has been determined previously), the location problem is equivalent to fitting a cone to the previously determined arrival times (for $3 \mathrm{D}$ scenarios, the fitting function is a hypercone). Although solving the location problem is not the purpose of this work, it is worth keeping in mind that the detection of hyperbolic patterns in data sets such as those shown in this work implicitly involves the identification of a cone as defined in equation 3 , which in practice is carried out by minimizing cost function 6 .

It is worth mentioning that, although arrival times are assumed to align along hyperbolas, they are actually on a cone for a $2 \mathrm{D}$ scenario, as shown in Figure 1b. Nonetheless, when a single vertical or slanted linear monitoring well is considered, a hyperbola is obtained
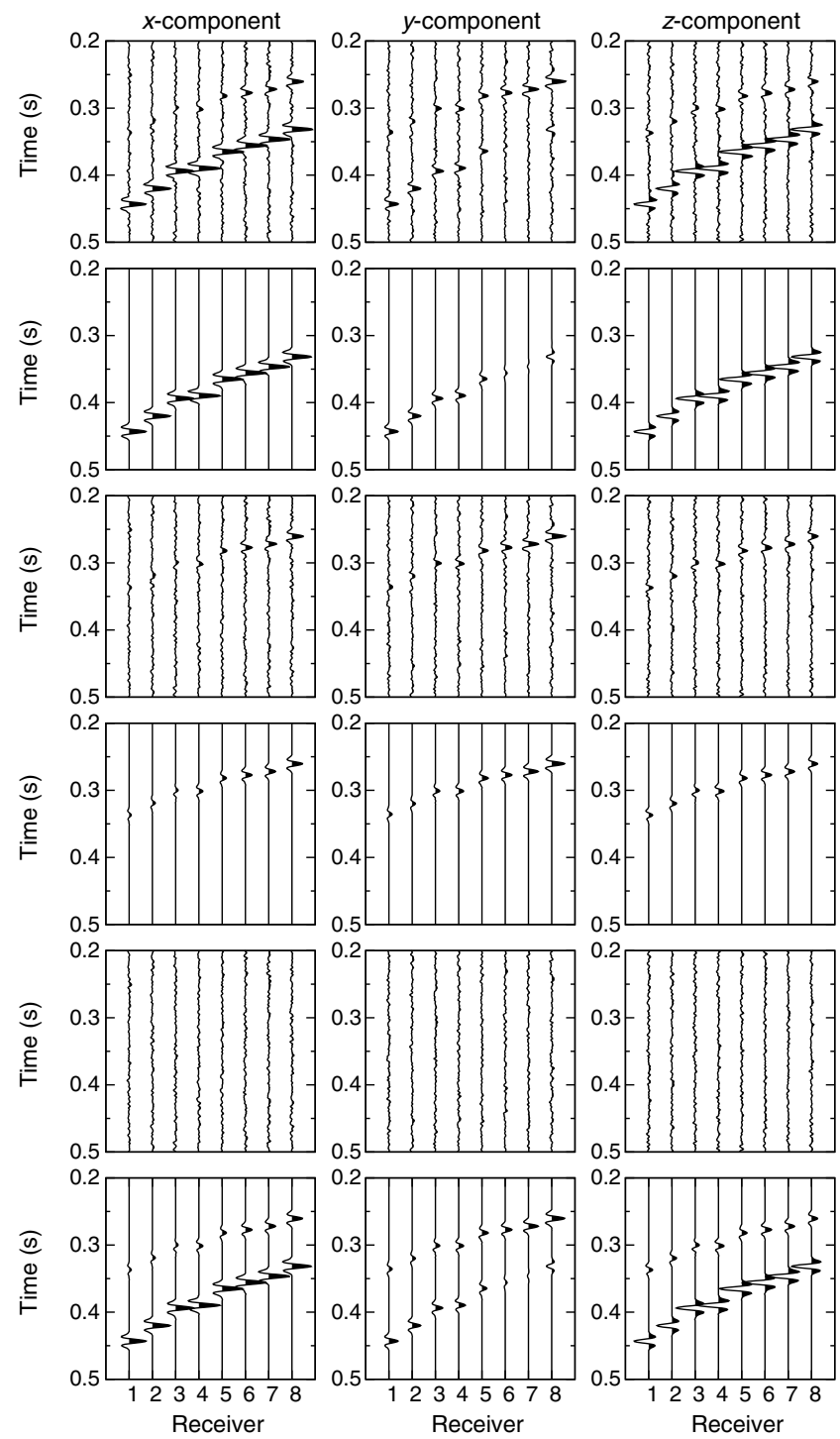

Figure 9. Microseismic signal denoising with synchronization through crosscorrelation and deflation (synthetic example 2). From top to bottom: (1) actual data, (2) first detected/denoised phase arrival, (3) residual, (4) second detected/denoised phase arrival, (5) residual, and (6) final denoised data. 
under the assumption of a constant velocity model. When the underlying velocity model is not constant, the geometric alignment of the arrival times is no longer valid. Still, an effective constant-velocity model coupled with a crosscorrelation-based synchronization procedure to align the individual wave arrivals can be used, as illustrated in Figure 9 for synthetic example 2. The synchronization process is required for the validity of the SVD filtering. Otherwise, the energy of the phase arrival will not be concentrated on the first eigenimages. One advantage of SVD filtering when coupled with an appropriate synchronization is that the resulting denoised data exhibit consistent waveforms throughout all the channels and components. In addition, the degree of filtering (and consistency) can be controlled by the number of eigenimages used in matrix approximation 9. The use of the first eigenimage only is very convenient from the computational point of view because its calculation is less expensive when using, for example, the so-called power methods (Antia, 2002; Burden and Faires, 2005). Nevertheless, because the dimensions of $S$ are usually very small, and the denoising is applied only once after detecting the phase arrival, the computation of the full SVD is not an issue for the current application.

In the "Results" section, we also compare the results of the proposed method with those obtained using the apex-shifted Radon approach (Sabbione et al., 2013), for the synthetic and field data examples. As shown by Sabbione et al. (2013), this technique is very effective for the detection of microseismic phase arrivals and to significantly enhance the $\mathrm{S} / \mathrm{N}$ of the signal. However, it is computationally more demanding than the method proposed in this work, especially for the 3D case because a large set of quadruplets (or quintuplets) needs to be scanned to compute the adjoint operator used in solving the inverse problem. Instead of a scan, the proposed method relies on a very efficient search using VFSA, which rapidly locates the most significant hyperbolic phase arrival within the analyzed data set. As for the accuracy of the results, in synthetic example 1, we show that except for the extreme case of $\mathrm{S} / \mathrm{N}=1$, they were comparable. In the very low $\mathrm{S} / \mathrm{N}$ case, the Radon approach results degraded significantly because the threshold used to restrict the signal support in the Radon domain was not able to isolate the hyperbolic phase arrival. In the field data example, we also show that the results were comparable in the lower $\mathrm{S} / \mathrm{N}$ cases. We do not apply the Radon technique for synthetic example 2 because the

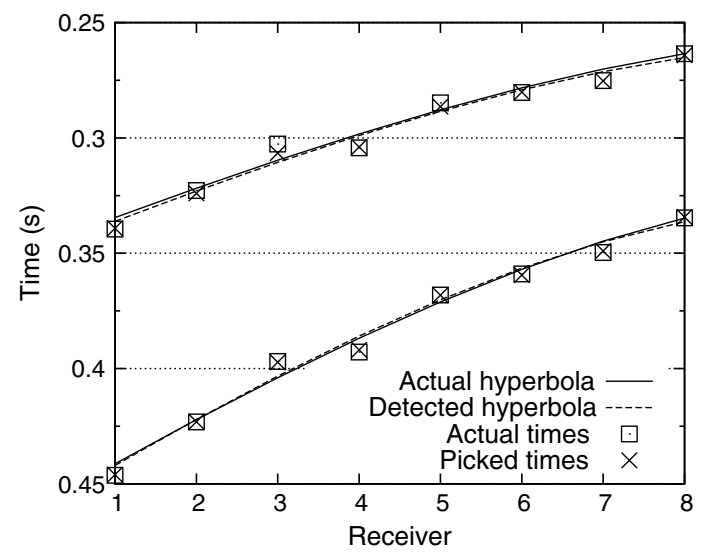

Figure 10. Actual and detected arrival times corresponding to the synthetic data as shown in Figure 9. phase arrivals were not aligned along hyperbolas and a synchronization scheme would have been necessary to stack the energy appropriately. Actually, the deflation and synchronization processes could have been incorporated into the Radon approach scheme, but this analysis is beyond the scope of this work.

Note that the proposed detection strategy is general and flexible in the sense that should a nonconstant velocity model be available, any ray-tracing algorithm could be used to compute the traveltimes that are subsequently used to calculate the coherency-energy measure. For simplicity, in the synthetic examples, we assume a $2 \mathrm{D}$ constant velocity model, which needs not be known a priori because it represents an unknown to be determined together with the parameters that define the corresponding hyperbola. In this context, the extension to 3D is straightforward. For these reasons, the so-called constant velocity hypothesis that leads to hyperbolic integration paths is not viewed as a limiting assumption of the proposed method, but as a means to derive rough estimates whenever the velocity model is unknown. When a velocity model is available, as in most actual microseismic processing projects, phase-arrival times should be computed using a forward modeling technique, and the proposed
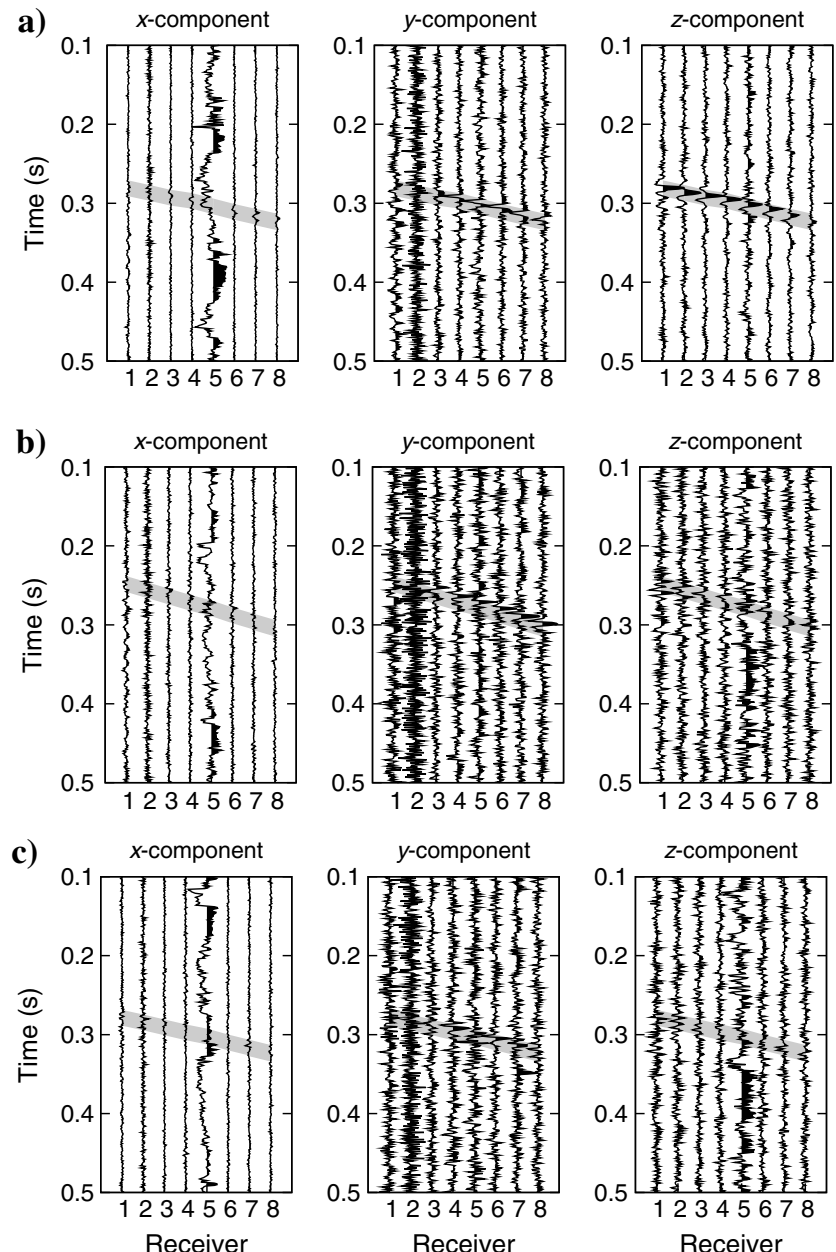

Figure 11. Field data: (a) moderate, (b) poor, and (c) very poor quality data. The shaded areas correspond to the final search window after the synchronization through crosscorrelation. For the processing, a 1-s time window was actually used. 
method could be applied after a slight modification of the cost function as described in the Synchronization section. Regarding the SA search ranges, these are not viewed as limiting assumptions either because they can be very large without significantly increasing the optimization complexity by virtue of the search capabilities of VFSA. In the synthetic examples, for instance, the search ranges were $x_{s}=0-1 \mathrm{~km}, z_{s}=1-2 \mathrm{~km}, t_{0}=0-0.2 \mathrm{~s}$, and $v=1-5 \mathrm{~km} / \mathrm{s}$. These ranges were wide enough so as to avoid adding unnecessary assumptions, but restrictive enough so as to preclude nonrealistic hyperbolic patterns.

In its current formulation, the deflation process only allows for the automatic detection of a single phase at a time within the search a)
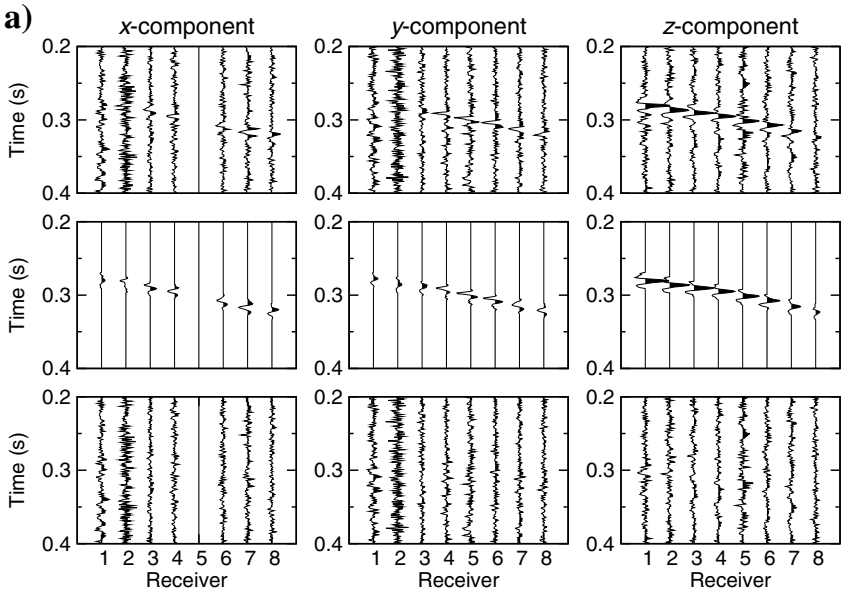

b)
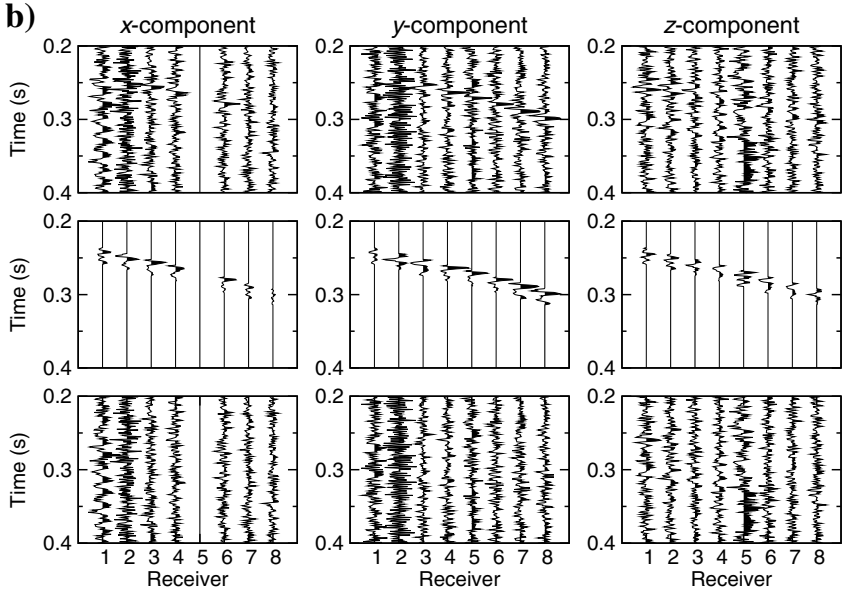

c)
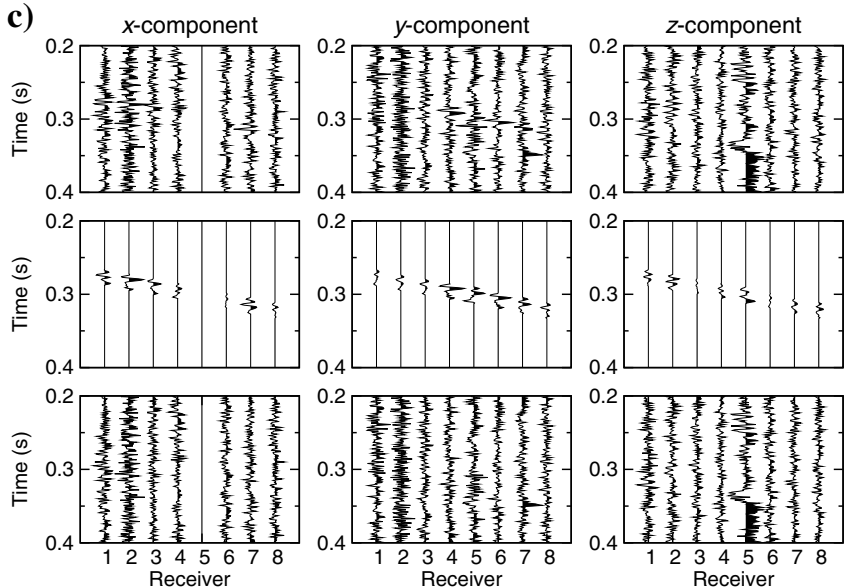
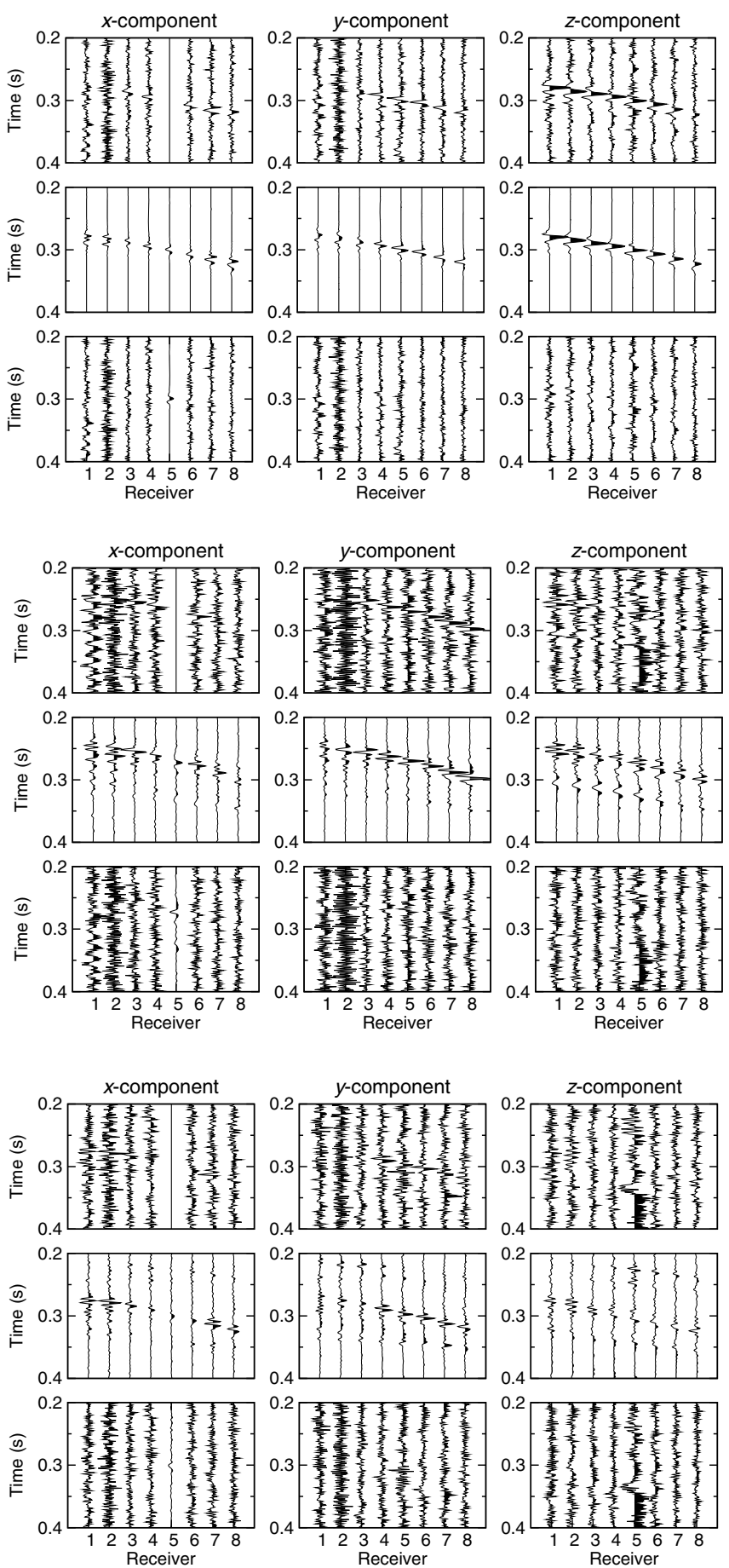

Figure 12. Microseismic signal denoising (field data example) using the proposed method (left column) and the hyperbolic Radon transform approach (right column): (a) moderate quality data, (b) poor quality data, and (c) very poor quality data. In each case, rows 1-3 represent the actual data, the detected/denoised phase arrival, and the residual, respectively. 
window (P- or S-wave splitting). This means that some extra work is required to identify the different detected phase arrivals and associate them with one or more microseismic events. There are various strategies that can be implemented to solve this problem. In the case that the $\mathrm{P}$ - and $\mathrm{S}$-wave velocity models are available, the method can be easily modified to search for two (or three) hyperbolas simultaneously (one for each phase arrival). To this end, one would have to redefine the coherence function $G$ as the sum of the coherence functions associated to each potential phase arrival. This implementation is straightforward. Another option would be to add extra unknowns to account for the P- and S-wave velocities under the constant effective velocity assumption, and do the same. Similarly, when there are two or more monitoring wells, there would be one hyperbolic pattern associated with each phase arrival and monitoring well. Then, the contribution of all the hyperbolic patterns would have to be added altogether into a single coherence function. The key difference is that the hyperbolas for each monitor well that are associated with the same phase arrival would share the same source coordinates and origin time.

\section{CONCLUSIONS}

The proposed technique allows one to automatically detect and denoise a microseismic phase arrival immersed in 3C noisy data. The technique is fast and accurate, provided a constant velocity model is appropriate to define the alignment of the arrivals along an hyperbola. For nonconstant velocity models, the proposed crosscorrelation-based synchronization process allows one to detect and appropriately denoise nonhyperbolic phase arrivals. In this context, SVD filtering promotes consistent waveforms throughout all the channels and components, independently of the regularity observed in the alignment of the time arrivals. Furthermore, a crosscorrelation-based $\mathrm{S} / \mathrm{N}$ indicator is provided to assess the significance of the reconstructed individual arrivals. This indicator could be used as a weighting factor for further processing and/or analysis of the microseismic data.

A deflation process is proposed to handle more than a single phase arrival within the analyzed time window. The deflation process is iterative, leading to a sequence of detected and denoised phase arrivals of decreasing energy that is repeated until the residual data contain no significant coherent energy. A simple energy-ratio confidence indicator that prevents the detection of false positives is provided for this purpose.

The detection-denoising strategy is very simple because the tuning parameters are just a few and easily set. These include the maximum number of VFSA iterations and the length of the time window associated with the hyperbola. In particular, we used 1000 iterations in all the examples for data windows of $0.5-1 \mathrm{~s}$ and a hyperbolic search window of 20-30 ms, just enough to encompass one period of the expected signal arrival.

Tests using synthetic and field data demonstrated that the proposed technique behaved very accurately when applied to moderate- to high-S/N data sets, and reasonably well when applied to very noisy data sets. The algorithm output includes the following: (1) estimates of the origin time, coordinates of the source, an effective velocity model, and the corresponding traveltimes, (2) consistent denoised wave arrivals throughout all the channels and components, and (3) two confidence indicators: one to asses the presence of an actual phase arrival, and the other to assess the reliability of the derived wave arrivals. Overall, the detection is robust to random noise, the derived traveltimes are accurate, and the denoised waveforms are consistent with the observed data.

\section{ACKNOWLEDGMENTS}

We thank the editor and the three reviewers for their comments and suggestions that helped to improve our manuscript. This work was partially financed by Agencia Nacional de Promoción Científica y Tecnológica, Argentina (PICT 2010-2129).

\section{REFERENCES}

Antia, H., 2002, Numerical methods for scientists and engineers 2nd ed.: Tata McGraw Hill.

Blias, E., and V. Grechka, 2013, Analytic solutions to the joint estimation of microseismic event locations and effective velocity model: Geophysics, 78, no. 3, KS51-KS61, doi: 10.1190/geo2012-0517.1.

Burden, R. L., and J. D. Faires, 2005, Numerical analysis 8th ed.: Thomson Brooks/Cole.

Chen, Z., and R. Stewart, 2006, A multi-window algorithm for real-time automatic detection and picking of P-phases of seismic events: CREWES Research Report, 18, 15.1-15.9.

Chopra, S., and K. Marfurt, 2007, Seismic attributes for prospect identification and reservoir characterization 1st ed.: SEG, Geophysical Developments 11.

DiCarlo, J. J., D. Zoccolan, and N. C. Rust, 2012, How does the brain solve visual object recognition?: Neuron, 73, 415-434, doi: 10.1016/j.neuron .2012 .01 .010 .

Eisner, L., B. Hulsey, P. Duncan, D. Jurick, H. Werner, and W. Keller, 2010, Comparison of surface and borehole locations of induced microseismicity: Geophysical Prospecting, 58, 809-820, doi: 10.1111/j.1365-2478 .2010.00867.x

Felleman, D. J., and D. van Essen, 1991, Distributed hierarchical processing in the primate cerebral cortex: Cerebral Cortex, 1, 1-47, doi: 10.1093/ cercor/1.1.1-a.

Galotti, K. M., 2014, Cognitive psychology: In and out of the laboratory (5th ed.): SAGE Publications, Inc.

Gharti, H. N., V. Oye, M. Roth, and D. Kühn, 2010, Automated microearthquake location using envelope stacking and robust global optimization: Geophysics, 75, no. 4, MA27-MA46, doi: 10.1190/1 .3432784 .

Golub, G., and C. Van Loan, 1989, Matrix computations 2nd ed.: Johns Hopkins University Press.

Gonzalez, R. C., and R. E. Wood, 2007, Digital image processing 3rd ed.: Prentice Hall.

Ingber, L., 1989, Very fast simulated re-annealing: Journal of Mathematical Computation and Modelling, 12, 967-973, doi: 10.1016/0895-7177(89) 90202-1.

Kendall, M., S. Maxwell, G. Foulger, L. Eisner, and Z. Lawrence, 2011, Special section. Microseismicity: Beyond dots in a box - Introduction: Geophysics, 76, no. 6, WC1-WC3, doi: 10.1190/geo-20111114-SPSEIN.1.

Lagos, S. R., J. I. Sabbione, and D. R. Velis, 2014, Very fast simulated annealing and particle swarm optimization for microseismic event location: 84th Annual International Meeting, SEG, Expanded Abstracts, 2188-2192.

Liu, J., and K. Marfurt, 2005, Matching pursuit decomposition using Morlet wavelet: 75th Annual International Meeting, SEG, Expanded Abstracts, 786-789.

Mallat, S., and Z. Zhang, 1993, Matching pursuit with time-frequency dictionaries: IEEE Transactions on Signal Processing, 41, 3397-3415, doi: $10.1109 / 78.258082$

Maxwell, S., 2014, Microseismic imaging of hydraulic fracturing: Improved engineering of unconventional shale reservoirs: SEG, Distinguished Instructor Series, 17

Maxwell, S. C., J. Rutledge, R. Jones, and M. Fehler, 2010, Petroleum reservoir characterization using downhole microseismic monitoring: Geophysics, 75, no. 5, 75A129-75A137, doi: 10.1190/1.3477966.

Michaud, G., and S. Leaney, 2008, Continuous microseismic mapping for real-time event detection and location: 78th Annual International Meeting, SEG, Expanded Abstracts, 1357-1361.

Munro, K., 2004, Automatic event detection and picking of P-wave arrivals: CREWES Research Report, 16, 12.1-12.10.

Neidell, N., and M. T. Taner, 1971, Semblance and other coherency measures for multichannel data: Geophysics, 36, 482-497, doi: 10.1190/1 .1440186 . 
Press, W. H., S. Teukolsky, W. Vetterling, and B. Flannery, 1992, Numerical recipes in FORTRAN: The art of scientific computing 2nd ed.: Cambridge University Press.

Sabbione, J., M. D. Sacchi, and D. R. Velis, 2015, Radon transform-based microseismic event detection and signal-to-noise ratio enhancement: Journal of Applied Geophysics, 113, 51-63, doi: 10.1016/j.jappgeo .2014 .12 .008

Sabbione, J. I., M. Sacchi, and D. Velis, 2013, Microseismic data denoising via an apex-shifted hyperbolic Radon transform: 83rd Annual International Meeting, SEG, Expanded Abstracts, 2155-2161.

Sabbione, J. I., and D. R. Velis, 2013, A robust method for microseismic event detection based on automatic phase pickers: Journal of Applied Geophysics, 99, 42-50, doi: 10.1016/j.jappgeo.2013.07.011.

Sacchi, M. D., 1998, A bootstrap procedure for high-resolution velocity analysis: Geophysics, 63, 1716-1725, doi: 10.1190/1.1444467.

Sun, J., L. Wang, and H. Hou, 2012, Application of micro-seismic monitoring technology in mining engineering: International Journal of Mining Science and Technology, 22, 79-83, doi: 10.1016/j.ijmst.2011.06.007.

Ursin, B., M. G. da Silva, and M. J. Porsani, 2014, Signal and traveltime parameter estimation using singular value decomposition: Geophysics, 79, no. 5, U1-U14, doi: 10.1190/geo2013-0260.1. van der Baan, M., D. Eaton, and M. Dusseault, 2013, Microseismic monitoring developments in hydraulic fracture stimulation, in R. Jefferey, ed., Effective and sustainable hydraulic fracturing: Intech.

Velis, D. R., J. I. Sabbione, and M. D. Sacchi, 2013, Fast and robust microseismic event detection using very fast simulated annealing: Presented at European Geosciences Union (EGU) General Assembly.

Vera Rodriguez, I., 2011, Automatic time-picking of microseismic data combining STA/LTA and the stationary discrete wavelet transform: Presented at CSPG CSEG CWLS Convention, Convention Abstracts.

Wang, Y., 2010, Multichannel matching pursuit for seismic trace decomposition: Geophysics, 75, no. 4, V61-V66, doi: 10.1190/1.3462015.

Yilmaz, O., 2001, Seismic data analysis: Processing, inversion, and interpretation of seismic data: SEG, Investigations in Geophysics.

Zhou, R., L. Huang, and J. Rutledge, 2010, Microseismic event location for monitoring $\mathrm{CO}_{2}$ injection using double difference tomography: The Leading Edge, 29, 208-214, doi: 10.1190/1.3304826.

Zimmer, U., and J. Jin, 2011, Fast search algorithms for automatic localization of microseismic events: CSEG Recorder, 36, 40-46. 